\title{
Correction to: Impact of Specimen Heterogeneity on Biomarkers in Repository Samples from Patients with Acute Myeloid Leukemia: A SWOG Report, by Pogosova-Agadjanyan, EL, et al. Biopreserv Biobank 2018;16(1):42-52. DOI: 10.1089/bio.2017.0079
}

In the February 2018 issue of Biopreservation and Biobanking (vol. 16, no. 1, pp. 42-52), in the article titled Impact of Specimen Heterogeneity on Biomarkers in Repository Samples from Patients with Acute Myeloid Leukemia: A SWOG Report by Era L. Pogosova-Agadjanyan et al., the authors identified several errors in the published article.

One of the errors was in Figure 2, on page 46, in which the images in the A and B panels were switched.

There was also a minor error on page 46, first column, dealing with the $p$ values for the BM and PB, although the interpretation remains the same. The following was corrected under the Results section: (BM: 95\% CI 1.2-2.6, $\boldsymbol{p}<\mathbf{0 . 0 0 1}$, PB: 95\% CI 0.4-2.6, $\boldsymbol{p}=\mathbf{0 . 0 0 9}$; Fig. 2).

The other error stemmed from a shift in columns in Supplementary Table S11 (the revised Suppl Table S11 has been reuploaded), which impacted a paragraph in the first column on Page 47, where the following changes were made:

BAALC, CEBPA, CD34, ERG1, EVII, KIT, and MN1 were significantly associated with normal cytogenetics in MNCs and AML blasts. Half of the biomarkers were significantly associated with normal cytogenetics in both unsorted MNCs and AML blasts, with RUNXI and JAG1 being significantly associated with normal cytogenetics in MNCs. CCNA, ERG1, GATA2, $I L 3 R A, R U N X I$, and $W T I$ were positively correlated with PB blast percentages in unsorted MNCs and AML blasts. EVII, FLT3, and KIT in unsorted MNCs and CEBPA in enriched blasts were significantly correlated with PB blast percentage. Similarly, BAALC, CCNA1, CD34, ERG1, IL3RA, KIT, and MN1 were significantly correlated with WBC in MNCs and blasts, whereas EVI1 was significantly correlated with WBC in unsorted MNCs only (Supplementary Table S11).

The online version has been corrected.

The authors apologize for these errors. 